\title{
Rectal Neuroendocrine Tumor in a Patient with
} CHEK2 Mutation

\author{
Shefali Khanna, MD', Deena Dahshan, MS4', Erin Fankhanel, MD', \\ Doreen Griswold, MD' ${ }^{1}$, Lynne J. Goebel, MD, FACP'
}

\section{ABSTRACT}

Author affiliations are listed at the end of this

We provide new information about CHEK2 mutation in association with a rectal neuroendocrine tumor (NET). A 67-year-old Caucasian woman diagnosed with estrogen receptor positive ductal carcinoma in situ breast cancer underwent genetic testing, revealing a CHEK2 mutation that prompted her to have a colonoscopy. This revealed a polyp with immunohistochemical staining positive for markers classically associated with NETs. Six-month follow up surveillance colonoscopy after initial polyp removal found no evidence of recurrence. While the CHEK2 mutation is known to be associated with adenocarcinomas, there is only one other reported case of its association with NETs.

article.

Correspondence to: Lynne J. Goebel, MD, FACP Marshall University Joan C. Edwards School of Medicine goebel@marshall.edu

\section{KEYWORDS}

Rectal carcinoid, CHEK2 mutation, rectal neuroendocrine tumor

\section{INTRODUCTION}

Discovery of a CHEK2 mutation in cancer patients necessitates discussion about the risk for additional tumors and screening to reduce morbidity and mortality from other concurrent CHEK2 mutation-related cancers. Rectal neuroendocrine tumors (NETs), formerly called carcinoids, are relatively rare, representing less than $1 \%$ of rectal cancers. ${ }^{1,2}$ While the CHEK2 mutation is associated with adenocarcinoma of the colon, it has not been identified in association with NETs, and, to our knowledge, there is only one other case of an appendiceal NET in a patient with CHEK2 mutation. ${ }^{3}$ We report a patient who had ductal carcinoma in situ (DCIS) breast cancer and a CHEK2 mutation and was later diagnosed with a rectal NET.

\section{CASE PRESENTATION}

A 67-year-old Caucasian female with a history of estrogen receptor-positive DCIS breast cancer had genetic testing with a panel of 28 actionable genes,

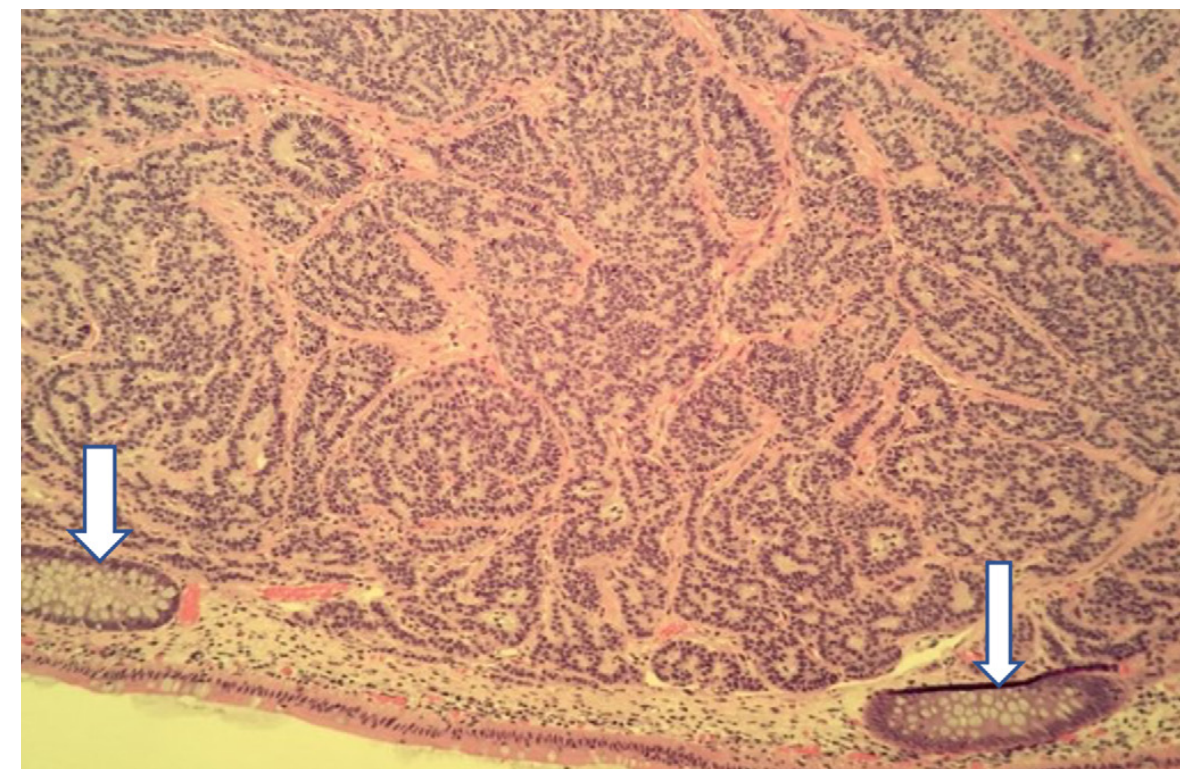

FIGURE 1. Low-power hematoxylin and eosin-stained image of the colonic mucosa with loss of the normal test tube appearance of colonic crypts due to distortion by the neoplasm. 100x magnification. The arrows indicate a normal crypt. 


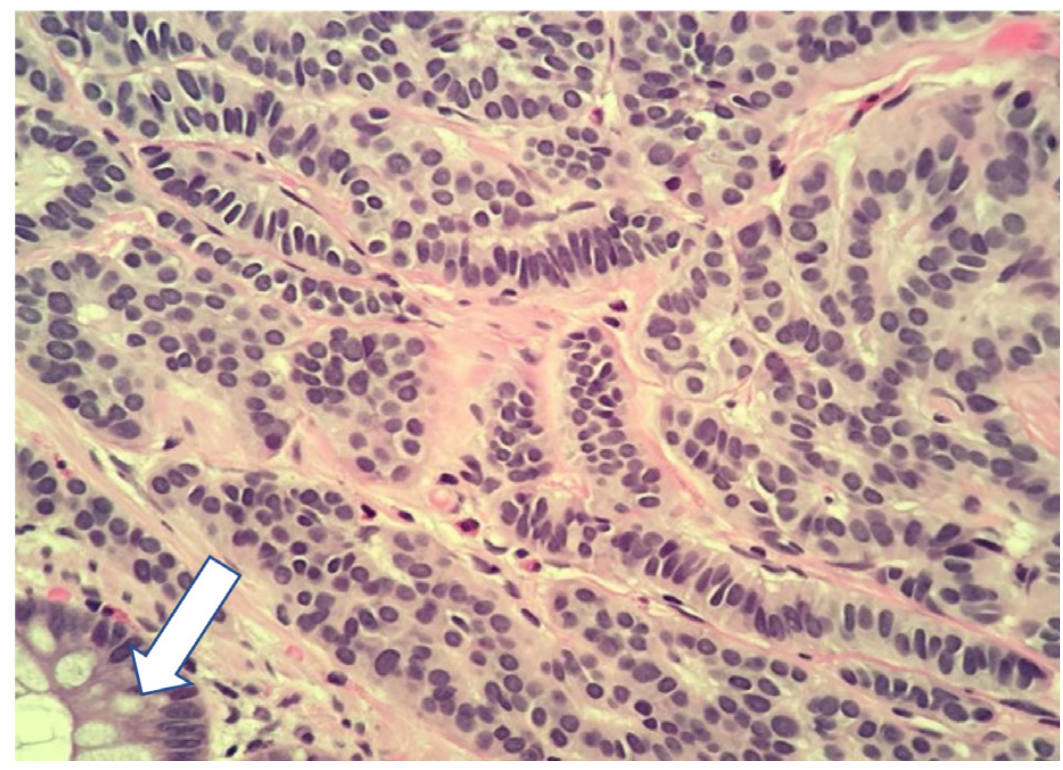

FIGURE 2. High-power hematoxylin and eosin-stained image of the neoplastic cells within the colonic submucosa. A portion of a normal colonic crypt (arrow) is present. 400x magnification.

including BRCA1 and BRCA2, as the result of a strong family history (her grandmother and sister) of breast cancer. The patient was found to have a germline $\mathrm{c} 1100$ del CHEK2 mutation. Because of the association of CHEK2 mutation with colorectal adenocarcinoma, she underwent colonoscopy. This was her first colonoscopy as previously, she had refused this procedure, opting instead for occult blood testing as a colorectal cancer screening. Upon colonoscopy, a polyp of less than $1 \mathrm{~cm}$ in diameter was discovered in the rectum. Pathology showed that the tumor was characterized by a submucosal neoplastic proliferation of cytologically bland, mitotically inactive cells arranged in cords and nests. The cells stained strongly positive for CD56, synaptophysin, and the cytokeratin cocktail AE1/AE3, which are markers classically associated with NETs (Figures 1-3). Patient consent was obtained for this case report.

The rectal tumor was completely removed by polypectomy during colonoscopy, and the location was subsequently tattooed to mark the tumor site. Surveillance for recurrence after 2 and 6 months found no evidence of recurrence.

\section{DISCUSSION}

CHEK2 germline mutation is known to be associated with adenocarcinomas of the breast and colon, but only one other case of NET associated with this mutation has been reported. ${ }^{3}$ Some providers offer next-generation genetic screening to all cancer patients without limitation by insurance or finances in order to develop a genetic registry. ${ }^{3}$ One such group uncovered six cases of NETs associated with different cancer-predisposing genes, including APC, MUTYH, MSH-6, MLH1, and CHEK2. Only one of the six patients harbored a CHEK2 mutation. This patient had an appendiceal goblet cell tumor, and immunohistochemical staining yielded a diagnosis of NET.

Gastrointestinal NETs are the most common type (43\%), and rectal location NETs account for only $15 \%$ of the gastrointestinal NETs. The second most common location for NETs is the lung, representing $31.9 \%$ of NET cases reported. ${ }^{4}$ Rectal and anal NETs with diameters of less than $1 \mathrm{~cm}$ have the best prognosis, so early detection is important. ${ }^{5} \mathrm{We}$ realize this report will not change practice, because we already perform colonoscopy on patients with CHEK2 mutation to look for adenocarcinoma; however, confirmation of the association of CHEK2

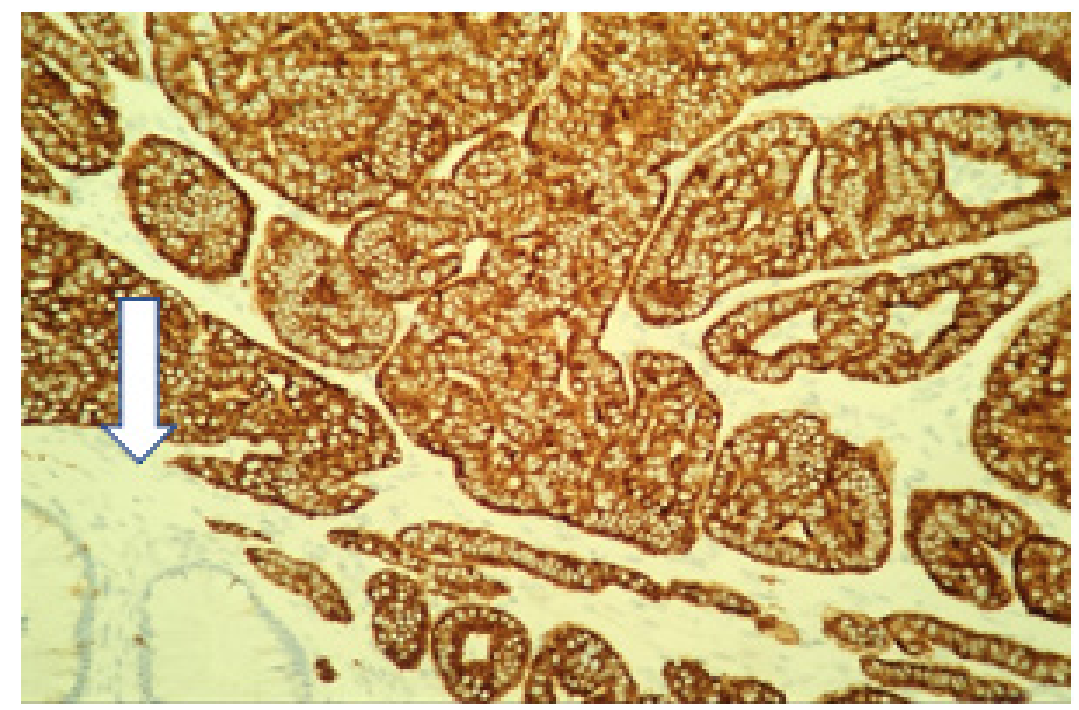

FIGURE 3. High-power image of the neoplastic cells stained with antibodies directed against synaptophysin, a marker of neuroendocrine differentiation. Normal colonic crypt is indicated by the arrow. 200x mds.marshall.edu/mjm

(c) 2021 Marshall Journal of Medicine
Marshall Journal of Medicine

Volume 6 Issue 1 
mutation with NETs may lead to trials of screening other parts of the body, such as the lung, for early detection and reduction of cancer mortality.

The CHEK2 gene codes for a protein that acts as a tumor suppressor, regulating excessive cell growth. In response to damage in the cell, CHEK2 communicates with other well-known regulators of mitosis as well such as tumor protein 53 (tp53)..$^{6}$ It is not surprising that a mutation that limits the action of CHEK2 results in tumor growth because the role of CHEK2 is to limit unwarranted proliferation. ${ }^{7}$

As more genetic testing is performed, we will likely identify additional tumors that are associated with CHEK2 mutation. For example, a 48-year-old female patient with the CHEK2 mutation was found to have adrenocortical carcinoma. ${ }^{8}$ Surveillance testing discovered an adenocarcinoma in her left lower lung 2 months later. Additionally, a populationbased study revealed an increased risk of breast cancer, stomach cancer, kidney cancer, sarcoma, and prostate cancer in patients with CHEK2 mutations. ${ }^{9}$ The NIH website reference to CHEK2 links this gene with prostate, breast, lung, colon, kidney, thyroid, and ovarian cancers as well as osteosarcoma. ${ }^{6}$

\section{CONCLUSION}

This study provides new information about CHEK2 mutation in association with a rectal NET, adding to the growing body of knowledge about this mutation. While CHEK2 mutations are associated with colonic adenocarcinomas, to our knowledge only one other reported case of CHEK2 mutation in association with a gastrointestinal NET has been reported. Greater knowledge of these associations may potentially lead to earlier diagnosis of cancer in patients with CHEK2 mutation, due to increased preventive screening in these high-risk patients. As more genetic testing is performed, we suspect there will be reports of additional cancers associated with CHEK2 mutation.

\section{CONFLICT OF INTEREST}

The authors declare that there is no conflict of interest.

\section{AUTHOR AFFILIATIONS}

1. Marshall University Joan C. Edwards School of Medicine, Huntington, West Virginia

\section{REFERENCES}

1. Ramage JK, Ahmed A, Ardill J, Bax N, Breen DJ, Caplin ME, Corrie P, Davar J, Davies AH, Lewington V, Meyer T, Newell-Price J, Poston G, Reed N, Rockall A, Steward W, Thakker RV, Toubanakis C, Valle J, Verbeke C, Grossman AB; UK and Ireland Neuroendocrine Tumour Society. Guidelines for the management of gastroenteropancreatic neuroendocrine (including carcinoid) tumours (NETs). Gut. 2012 Jan;61(1):6-32.

2. ASCO foundation. Cancer.Net. Accessed 24 August 2019. https://www.cancer.net/cancertypes/neuroendocrine-tumors/introduction

3. Kidambi TD, Pedley C, Blanco A, Bergsland EK, Terdiman JP. Lower gastrointestinal neuroendocrine neoplasms associated with hereditary cancer syndromes: A case series. Fam Cancer. 2017 Oct;16(4):537-543.

4. Kojima M, Ikeda K, Saito N, Sakuyama N, Koushi K, Kawano S, Watanabe T, Sugihara K, Ito M, Ochiai A. Neuroendocrine Tumors of the Large Intestine: Clinicopathological Features and Predictive Factors of Lymph Node Metastasis. Front Oncol. 2016 Jul;6:173.

5. André TR, Brito $M$, Freire JG, Moreira A. Rectal and anal canal neuroendocrine tumours. J Gastrointest Oncol. 2018 Apr;9(2):354-357.

6. U.S. National Library of Reference Writers. Chek2 Gene. Accessed 6 August 2019. https://ghr.nlm. nih.gov/gene/CHEK2\#conditions.

7. Suchy J, Cybulski C, Wokołorczyk D, Oszurek O, Górski B, Debniak T, Jakubowska A, Gronwald J, Huzarski T, Byrski T, Dziuba I, Gogacz M, Wiśniowski R, Wandzel P, Banaszkiewicz Z, Kurzawski G, Kładny J, Narod SA, Lubiński J. CHEK2 mutations and HNPCC-related colorectal cancer. Int J Cancer. 2010 June 15;126(12):3005-9.

8. Xi C, Tanakchi S, Raygada M, Davis JL, Rivero JD. Case Report of an Adrenocortical Carcinoma Associated with Germline CHEK2 Mutation. J Endocr Soc. 2018 December 12;3(1):284-290.

9. Näslund-Koch C, Nordestgaard BC, Bojesen SE. Increased Risk for Other Cancers in Addition to Breast Cancer for CHEK2*1100delC Heterozygotes 
Estimated from the Copenhagen General

Population Study. J Clin Oncol. 2016 April

10;34(11):1208-16. 